\title{
THE TREATMENT OF INGUINAL HERNIA IN MALE INFANTS.
}

\author{
By R. A. RAMSAY, M.Ch.(Cantab.), F.R.C.S.Eng. \\ Senior Surgeon to the Metropolitan Hospital; Senior Surgeon to the Belgrave Hospital for \\ Children; Surgical Consultant L.C.C. Hospital Service.
}

Hernia in infants is not an uncommon condition; discovered at birth or during the early months of life it surprises and often greatly distresses the parents of the patient so that advice is usually sought soon after it is noticed. The advice to be given and the treatment to be adopted require careful consideration, since the condition occurring in an infant shews several important peculiarities, and if a satisfactory result is to be obtained these must be kept in mind when dealing with the case.

In an adult a hernia is treated on account of the inconvenience and discomfort associated with it and because of the risk of strangulation. In an infant the risk of strangulation is very remote; the tissues are so soft that it is extremely rare for true irreducibility to occur, and in practically every case non-operative measures will overcome any difficulty in reduction. Also, the inconvenience has not the importance that it has in an adult; there is no occupation to be interfered with and in this respect the mother is chiefly affected since extra care and attention are required. It must not be forgotten that a hernia, especially a large one that is continually coming down, causes discomfort and crying; the child may not rest, may not take its food quietly and may not thrive so that measures must be taken to prevent the continued protrusion of abdominal contents. But a hernia in an infant if not completely and permanently cured will persist and as the patient grows older will sooner or later be an inconvenience and even, from the risk of strangulation, a danger; so that it is advisable to take such measures as are necessary to prevent this persistence. If this is done the child can, when old enough, commence his education without fear of interruption or loss of time from this cause, and in adult life will be able to lead a normal and active life without the inconvenience and disabilities with which the "ruptured" have to contend.

The methods of treatment which have to be considered are two, namely:the application of a truss, and the operation for radical cure; these are the same methods that may be used in the case of an adult, but if detailed consideration is given to the peculiarities of hernia in infants and of its treatment it will be seen that the problem is not exactly similar.

\section{Treatment by a Truss.}

The truss, as is well known, is an appliance which, by exerting pressure upon the inguinal canal and the hernial sac, compresses the neck of the latter and prevents the passage of abdominal contents through the layers of the abdominal muscles. In an adult this is all that a truss is expected to do; as long as it is properly fitted and in a satisfactory state of repair it will prevent the hernia from coming down, but it is now almost universally admitted that a truss will not cure it. In other words the neck of the sac will never become obliterated and at any time, when the truss is not in position, a strain or cough may cause the swelling to reappear. But in a child, whatever may be the case in an adult, the presence of hernia means that a developmental process is delayed; the processus vaginalis which should have become shut off at its origin from the parietal peritoneum has not become so, leaving 
a sac of greater or less length communicating at the internal ring with the abdominal cavity, and it is into this tubular space that the hernia passes, provided that some part of the abdominal contents have sufficient freedom of movement to do so and that the abdominal pressure is raised by straining or crying sufficiently to force this mobile part through the neck. Since many developmental processes, even when delayed, continue to progress and become more or less complete after birth, it is not unreasonable to suppose it possible for the processus vaginalis to become shut off at some time in the early months of life, provided that it is not repeatedly dilated and so kept patent by the frequent passage into it of a hernial protrusion. On this supposition a truss may, by continually preventing over a considerable period the extrusion of abdominal contents, give an opportunity for the closure of the patent processus vaginalis. A somewhat parallel condition is seen in hydrocele of the cord in infants in a great many cases disappearing after several tappings. The fluid had prevented the walls of the vaginal process, in this case shut off from the abdomen, but not reduced at birth to normal dimensions, from completing their closure and leaving only the usual sac investing the testicle.

Thus a truss is applied to an infant not only as a palliative measure but also with some hope of cure, and to fulfil these objects it must be comfortable and clean and it must efficiently control the neck of the sac so that no protrusion occurs; if these ideals are not attained the truss is not only useless but causes great inconvenience. To control the neck of a hernial sac the pad of the truss must press the anterior wall of the inguinal canal firmly against the posterior wall, especially over the internal ring where the sac communicates with the abdominal cavity; this pressure must be directed backwards, since that is the only way in which the inguinal canal and its contained sac can be compressed. The pressure must be light when there is no tendency for the hernia to come down, but must increase as soon as any tendency to bulge at the internal ring occurs. This varying pressure is necessary for comfort and efficiency and is only attainable by the use of a spring taking its fixed point on the sacrum behind, correctly shaped so as to fit the body and bring the pad over the desired area, and with a spring of the correct tension so that it does not cause discomfort or too firm pressure when the patient is quiet, but strong enough to give increasing resistance as soon as any bulging. of the abdominal wall or inguinal canal causes the pad to be pushed forward. In fitting a truss to an infant, the spring has to be of exactly the right tension; many such springs are too strong and cause discomfort, pain and even injury to the delicate skin of the patient; on the other hand, when the truss is first applied it is advisable to make the patient cry so as to see if the resistance of the spring is sufficient to control the hernia. The truss should be of the correct shape and should lie on the sacrum behind, pass between the crest of the ilium and the great trocanter and have the pad over the situation of the inguinal canal; if the hernia passes into the scrotum the continuation known as a rat-tail should be added, and this rat-tail should be a soft pad and should not, as in some trusses, have any metal incorporated in it. The truss should be covered with rubber so that the baby may be bathed with it on and that it may easily be kept clean. It may be ordered half an inch larger than the actual measurement round the body, in the line where the spring should lie, to allow for growth in cases where expense is an object, the extra girth being at first taken up by the button and later let out as the patient grows. Lastly the truss must be well made; the rubber covering must be good and the spring must be of the best quality so as to avoid the trouble and expense of breakage. A word of warning must be given on the subject 
of girdles, elastic or rigid, with a pad over the inguinal canal. The principle of such appliances is incorrect since the pressure is applied all round the body and any bulging is met by an increase of this circular compression and not by the necessary backward pressure over the inguinal canal.

The theory of cure by truss is so tempting, and, if true, this form of treatment is so obviously the best, that the writer over a period of several years treated a considerable number of patients by this method. To each case that had a sufficiently intelligent mother a truss was applied till the age of two, careful enquiry being made as to whether the hernia had ever been down; if there had been any such protrusion the case was considered as unsuccessful. At the age of two, when, if ever, the closure of the neck should be complete, the truss was left off altogether, and the patient was kept under observation for a further two years. If in that time the hernia had never been down, as happened in a fair proportion of cases, the treatment was considered successful. But was it? A certain number of patients came to operation after treatment by trusses and the writer was thus able to a certain extent to verify the results. Thus in the same week two patients who had been treated by trusses for double hernia were brought for advice towards the end of the final year of observation because a hernia had come down on one side. As these cases were obvious failures, and since the continuance of the developmental process of closure could hardly be hoped for any longer, operation was advised and permission obtained to explore the other side on which no recurrence had been seen; as was expected the sac was still present where the hernia had recently been down, but in both cases a patent sac was also found on the apparently sound side where no protrusion had taken place since the early months of life. These two cases prove that one cannot count on obliteration of the neck of a hernial sac by the long continued and apparently successful use of a truss. On the other hand, in a few cases where a truss has been worn apparently successfully on one side only, a hernia has appeared on the sound side at such a late age as not to justify any treatment other than operative; when possible permission to explore the side where the truss was worn has been obtained and in a certain number of these no sac has been found, its place being taken by a fibrous cord reaching to the parietal peritoneum.

These cases shew that while in some cases a truss produces a cure in accordance with the theory stated above, in others it certainly does not do so and that it is impossible to tell by mere clinical examination what has really happened. So that in such children it must always be considered as doubtful whether a potential hernia which may appear in later childhood, in youth or even in adult life, is present or not. In view of this uncertainty the writer felt forced to abandon treatment by truss with a view to curing a hernia in an infant.

\section{Radical Cure by Operation.}

The operation for radical cure of hernia in an infant has one essential object, the same as that aimed at by the use of a truss, namely the closure of the neck of the sac. It is true that the rest of the sac is removed, but this is to prevent the possible formation of a hydrocele in it later, rather than to influence the radical cure of the hernia. In the adult it is usual to strengthen the walls of the inguinal canal by suturing the muscular layers in various positions according to the particular operation chosen. But in a small child the muscles have not been displaced by the frequent protrusion of the hernia over a period of many years, and they fall into their normal position after they have been drawn aside during the 
exposure of the neck of the sac. It is usually only necessary in an infant to suture the incision of the external oblique where it has been divided in opening the inguinal canal. An exception may be made in the case of a large scrotal hernia which has continued to remain down in spite of trusses and has so formed for itself a large gap between the muscles; here it is quite reasonable to bring down the conjoined tendon to Poupart's ligament by a few sutures as is usual when the operation is performed in an adult. After such an ideal operation the patient should be cured once and for all so that no fear of recurrence in later life with its inconvenience or even danger need be entertained.

Although such an operation sounds simple there are, when it is performed in an infant under a year old, certain difficulties and dangers which prevent the ideal as described above from always being attained. It is on the whole wiser in general not to operate on a small baby for any reason if it is possible to wait till a rather later age; while these infants can and do stand operations, done rapidly and gently, very well, their tender age is undoubtedly an added risk. It is not always easy to keep a baby clean, when the area of operation is likely to be soiled frequently by urine or fæces; this can be overcome by careful nursing, which is made easier by omitting a dressing of any sort; but an advantage is certainly gained if an inguinal operation can be deferred until habits of cleanliness are more established. These points are not of themselves of the first importance but they cannot be overlooked when considered in association with more serious difficulties.

It has been said that the neck of the sac must be found and ligatured, and to do this it must be separated from the normal constituents of the spermatic cord; but in a small infant the sac is a very thin and delicate structure not always easily seen, and in separating it, its wall may easily be torn; should such a tear extend to the neck it is extremely difficult to find the edges of the sac and so make sure of including the whole of the neck in the ligature; if an opening should be left the hernia is almost certain to recur and cause great disappointment, while a second operation for closure of the aperture is far from easy. This delicacy of the sac and its liability to tear account for the fact that recurrence is not uncommon after an operation for radical cure at a very early age.

Another danger in such an operation is that of injury to the vas deferens. This structure is extremely small, and although it can always be felt if carefully sought for, it must be handled with the greatest gentleness for a pinch with forceps may be sufficient to obliterate its lumen; therefore, in separating the coverings from the spermatic cord, these coverings and the hernial sac should be peeled off the vas, and the vas itself should never be touched with the forceps.

Lastly, it occasionally happens that an incompletely descended testicle associated with the hernia may, in early infancy, lie actually at the internal ring and hamper by its presence the isolation and ligature of the sac at this point; if the testicle can be pulled down, the ligature, though difficult, is possible; but, if, as is sometimes found at an early age, the testicle will not move so as to free the neck the satisfactory cure of the hernia is impossible without prejudicing the future migration, either natural or operative, of the testicle.

If these points are considered it will be realized that early operation for radical cure of hernia in infants is attended by difficulties such as seriously to prejudice the result. If, however, the operation can be postponed until some 
time in the third year of life, it will be found that these difficulties will largely have disappeared. The child is older; habits of cleanliness are more established; the parts are larger and less delicate and therefore the neck of the sac can be more certainly isolated without tearing; and the vas is more easily distinguishable and therefore less likely to be injured.

After some years of experience of the treatment of hernia by truss on the one hand and by operation at an early age on the other, the writer has come to the conclusion that a combination of the two methods gives, in his hands at any rate, the most satisfactory results. A properly fitting, well made truss is applied continuously till as late as possible in the third year of life; the truss must be a good one, the extra expense will be a saving in the end for a good truss will fit, will be comfortable and will not break. The truss must be replaced periodically as the child grows, but if ordered rather large each time these replacements will be reduced to a minimum. When the time comes radical cure as described above is performed; and it is well to obtain permission to operate on both sides even if no hernia has ever been seen in the apparently normal groin; the surgeon who does this as a routine will be surprised at the number of times such a procedure will enable him to remove a hernial sac and so prevent the appearance of a hernia there at some later date. It may be objected that the prolonged wearing of a truss may produce fibrosis as is often the case in adults; but in an infant a truss with a spring of the correct strength does not do so.

There are certain contra-indications to this plan of treatment. When there is an unintelligent mother, when there are many other small children or when the home conditions are poor, the care of the truss will almost certainly be unto satisfactory, and also the question of expense may be of great importance ; in such a case the surgeon will be almost forced to operate early in spite of the unsatisfactory nature of the procedure. Some parents, usually those to whom expense is less important and who can have necessary care given to the baby, are nervous and distressed at the abnormal condition and urge the surgeon not to delay operation; in these cases an attempt should be made to persuade them to agree to delay and this is often a matter of some difficulty. Lastly, the presence of an incompletely descended testicle may introduce complications; the age of the patient and the position of the testicle as well as its progress towards its proper situation must be carefully considered in each case and a compromise found which will give the best possible result to each of the associated conditions.

There is a great lack of uniformity in the methods of treating inguinal hernia in male infants and the subject is passed over very briefly in many text books. As the writer of this paper has been greatly interested in the question and has come to certain conclusions and settled on a fairly uniform line of treatment it is felt that the description of this and the reasons for its adoption may not be without interest. Briefly summed up the method of treatment advised consists in the application of a properly fitting and well constructed truss until some time in the third year of life when an operation for radical cure is performed on both sides, even when a hernia has only been seen on one side. Thus the hernia is controlled and its effects largely eliminated during the early months when operation is neither easy nor satisfactory; then at a more suitable age the radical cure is performed with every hope of success. In this way the ideal result is obtained of a permanent and complete removal, before the more active part of life begins, of an inconvenient and even dangerous condition. 